\title{
PERCEPÇÃO E ELASTICIDADE DE PREÇO PARA EMBALAGENS SUSTENTÁVEIS DE ALIMENTOS
}

\author{
Laura Moura Kohmann* \\ Janine Fleith de Medeiros ${ }^{* *}$ \\ Gabriel Vidor*** \\ José Luis Duarte Ribeiro*****
}

RESUMO: As embalagens, principalmente de alimentos, representam grande parte dos resíduos sólidos gerados. Nesse sentido, o presente artigo buscou verificar, através de pesquisa descritiva, a intenção de compra de alimentos com embalagens sustentáveis, seja essa sustentabilidade obtida através de embalagens biodegradáveis, de reciclagem ou da aplicação da logística reversa. Resultados preliminares indicam que essa tendência é particularmente acentuada no caso de mulheres jovens e com nível elevado de escolaridade. A intenção de compra diminuiu em todos os grupos quando o alimento com embalagem sustentável apresentava custo acima do preço de mercado, revelando que a tolerância a acréscimos no preço é, em média, de 10\%. $\mathrm{O}$ investimento de empresas em sustentabilidade, portanto, causa impactos positivos na decisão de compra do consumidor, principalmente nos que estão abaixo dos 50 anos.

PALAVRAS-CHAVE: Comportamento do consumidor; Embalagens de alimentos; Sustentabilidade.

\section{PERCEPTION AND ELASTICITY OF PRICES FOR SUSTAINABLE FOOD PACKAGES}

ABSTRACT: Packing constitutes the majority of solid wastes produced, especially in the case of food. Current research verifies by descriptive analysis the intention of

Especialista em Desenvolvimento de Produtos e Gestão na Indústria de Alimentos pela Universidade de Passo Fundo (UPF), Brasil.

** Doutora em Engenharia de Produção pela Escola de Engenharia da UFRGS; Docente nos cursos de graduação em Engenharia de Produção e Engenharia de Alimentos e de Pós-graduação Stricto sensu em Administração na Universidade de Passo Fundo (UPF), Brasil; E-mail: janine@upf.br

*** Doutor em Engenharia de Produção pela Universidade Federal do Rio Grande do Sul (UFRGS), Brasil; Docente Adjunto I na Universidade de Caxias do Sul (UCS), Brasil.

**** Doutor em Engenharia pela Universidade Federal do Rio Grande do Sul (UFRGS); Docente Associado da Universidade Federal do Rio Grande do Sul (UFRGS), Coordenador do Programa de Pós-graduação em Engenharia de Produção, Brasil. 
purchasing food wrapped in sustainable packages, whether the sustainability issue is foregrounded on biodegradable packages, or on recycling or on the application of reverse logistics. Preliminary results show that trend is specially underscored in the case of young women with high education level. Purchase intention decreases in all groups when sustainable package features costs above market prices, with an average $10 \%$ tolerance in price rise. Firms' investment in sustainability causes positive impacts on consumers' purchase decision, especially on people under 50 years.

KEY WORDS: Sustainability; Consumer's behavior; Food packages.

\section{INTRODUÇÃO}

Antes da Revolução Industrial do século XVIII, a produção de resíduos se resumia basicamente em resíduos orgânicos, não causando impacto severo no meio ambiente. A partir deste marco histórico, iniciou-se o chamado processo de urbanização, cuja maior característica é o consumismo (CAVALCANTE, 2002; DIAS, 2002 apud HISATUGO, 2007) aliado ao uso intenso de recursos naturais.

A exploração aguda dos recursos naturais, que ocorria indiscriminadamente até algumas décadas atrás, conduziu à grande preocupação com o meio ambiente observada nos dias atuais. No entanto, essa exploração também contribuiu para a formação de uma cultura ainda relutante em aceitar as mudanças de hábitos necessárias para estagnar os estragos causados pelo consumismo (KASSARGIAN, 1971; DINATO, 1999 apud BEDANTE, 2004; KOLLER et al., 2001).

Apesar disso, de forma gradativa, os consumidores estão percebendo o impacto que seus hábitos de consumo causam ao meio ambiente (AKENJI; BENGTSSON, 2010; WATKINS et al., 2015; ZHAO et al., 2014). Isso bastou para que muitas empresas, visando alcançar o consumidor ecologicamente consciente, identificassem um nicho de mercado promissor e passassem a oferecer produtos com menor impacto ambiental, utilizando desse diferencial para conquistar uma fatia maior de mercado (MacDONALD; SHE, 2015).

Neste sentido, o objetivo geral da pesquisa de mercado apresentada neste artigo centrou em verificar a percepção dos consumidores frente a produtos 
alimentícios que possuam embalagem sustentável. Pontualmente, buscou-se conhecer a elasticidade de preço possível para produtos com tais características, bem como se há interferência de características demográficas para a predisposição de compra.

\section{SUSTENTABILIDADE EM EMBALAGENS}

Em 1987 a Comissão Nacional do Meio Ambiente das Nações Unidas realizou um estudo no qual se introduziu o conceito de sustentabilidade. Este estudo, conhecido como Relatório Brundtland, define sustentabilidade como sendo "o desenvolvimento que satisfaz as necessidades atuais sem comprometer a habilidade das futuras gerações em satisfazer suas necessidades" (WORLD COMMISSION ON ENVIRONMENT AND DEVELOPMENT, 1987). Segundo Siche et al. (2007), a palavra sustentabilidade tem sua origem no latim, sustentare, ou seja, conservar em bom estado, manter. Conforme Elkington (1987), sustentabilidade refere-se ao alcance de uma vantagem competitiva equilibrada em um tripé que contempla pilares econômico, ambiental e social. Assim sendo, embora seja corrente a necessidade empresarial de sustentabilidade econômica, é fundamental que as organizações também busquem e promovam práticas de sustentabilidade social e ambiental.

Aproximadamente 30\% dos resíduos sólidos gerados vêm das embalagens, que em quase sua totalidade são fabricadas a partir de fontes não renováveis e não biodegradáveis, como os plásticos poliméricos (WALDMAN, 2011). Apesar da contribuição dos resíduos plásticos ser pequena em comparação a resíduos orgânicos, seu aspecto negativo está no longo tempo que esse material leva para se decompor, variando de 100 a 450 anos (ARAÚJO, 2014).

$\mathrm{Na}$ sua maioria, as embalagens de alimentos não envolvem apenas um tipo de material. Usualmente, são utilizados papelão, alumínio, diferentes tipos de polímeros, além das tintas e vernizes das embalagens externas e dos resíduos de alimentos nas embalagens que foram descartadas. Além disso, há a geração de resíduos de embalagem antes e depois do processo de industrialização, pois existem ainda as embalagens das matérias-primas que chegam à indústria de alimentos para então serem processadas (CARR, 2007). 
Neste cenário, destaca-se que uma tentativa de diminuir o efeito negativo das embalagens de alimentos centra no desenvolvimento de polímeros biodegradáveis feitos a partir de matéria-prima agrícola, como o amido, que forneçam propriedades semelhantes aos polímeros, com a vantagem da biodegradação no meio ambiente (CARR, 2007).

Coutinho et al. (2004) compararam plásticos biodegradáveis com plástico convencional e concluíram que os biodegradáveis são menos aplicáveis em função de sua baixa flexibilidade e custo mais elevado; contudo, o benefício da biodegradabilidade ainda capta a atenção de empresas que identificam a importância de oferecer um produto com diferencial ecológico.

Até a década de 90, a aplicação de plásticos reciclados em embalagens alimentícias era proibida em todo o mundo (SANTOS et al., 2004), cuja maior utilização era em objetos de utilidade doméstica, como enchimento de almofadas, tecidos e recipientes, desde que não utilizados para produtos alimentícios (SPINACÉ; DE PAOLI, 2001). As embalagens poliméricas mais utilizadas em alimentos são as chamadas termoplásticas e, em menor volume, as termofixas. Na reciclagem das embalagens termoplásticas, é necessário realizar uma etapa de caracterização e separação dos contaminantes que podem estar presentes nestas embalagens. Estas etapas anteriores à reciclagem em si podem aumentar os custos desta atividade consideravelmente (FORLIN; FARIA, 2002).

No Brasil, a quantidade aplicada de polímero reciclado em embalagem de alimentos é restringida quando a mesma entra em contato com o alimento, sendo apenas liberado o uso para embalagens secundárias ou terciárias ou em embalagens multicamadas; nos Estados Unidos, embalagens PET recicladas podem ser utilizadas em contato direto com alimentos (SANTOS et al., 2004; SPINACÉ; DE PAOLI, 2004).

A portaria ${ }^{0} 987$ do Ministério da Saúde, publicada em 1998, autoriza que o PET reciclado seja aplicado em embalagens multicamadas de bebidas carbonatadas. A maioria do PET reciclado no Brasil é destinada à produção de fibra têxtil (41\%) e o restante é convertido em cordas, mantas de não tecido, resinas insaturadas, cerdas de vassouras e embalagens de produtos não alimentícios (SPINACÉ; DE PAOLI, 2004).

Outro modo de trazer sustentabilidade às embalagens de alimentos é a aplicação da logística reversa. Como o próprio nome diz, trata-se de a embalagem fazer o caminho oposto à fabricação, com a possibilidade de reintegrar seus 
componentes à cadeia de produção. De acordo com os princípios de marketing, ela pode ser definida como "o movimento de mercadorias de um consumidor para um produtor em um canal de distribuição" (BRITO; DEKKER, 2002). A aplicação deste método é motivada pelos fatores de economia, pela redução de custos de operação, de legislação ambiental, de acordo com a Política Nacional de Resíduos Sólidos e de responsabilidade estendida, que diz respeito à responsabilidade da empresa com o ciclo de vida do produto (GONÇALVES-DIAS; TEODÓSIO, 2006).

Para analisar a cadeia de logística reversa, três perguntas devem ser feitas: por que, o que e como. Isso implica analisar porque é necessário proceder com o retorno das embalagens, quais são as características destas embalagens que estão retornando e como esse retorno é feito (BRITO; DEKKER, 2002; GONÇALVES-DIAS; TEODÓSIO, 2006).

\section{MÉTODO}

Tendo por referência o objetivo do presente estudo, que centrava verificar a predisposição de compra dos consumidores frente a produtos alimentícios que possuam embalagem sustentável, foi desenvolvida uma pesquisa descritiva, de abordagem quantitativa e, segundo o procedimento técnico, um levantamento. Os estudos descritivos possuem como objetivo primordial a descrição de determinadas características da população. A abordagem quantitativa caracteriza-se pela quantificação tanto na coleta quanto no tratamento dos dados, fato que possibilita uma margem de segurança maior quanto às inferências. Por fim, as pesquisas de levantamento caracterizam-se pelo questionamento direto das pessoas cujo comportamento se deseja conhecer (MALHOTRA, 2006).

A amostra caracteriza-se por ser não probabilística estratificada. Não probabilística porque não foi considerada a variabilidade da população na seleção da amostra, e estratificada porque os respondentes foram divididos por gênero, faixa etária (21 a 30 anos, 31 a 40 anos, 41 a 50 anos, 51 a 60 anos e 61 a 70 anos), escolaridade (ensino médio, graduação e pós-graduação) e renda mensal (até $R \$ 1.500,00$, de $R \$ 1.501,00$ a $R \$ 3.000,00$, de $R \$ 3.001,00$ a $R \$ 5.000,00$ e acima de $R \$ 5.001,00)$. O procedimento de coleta de dados foi realizado apenas por uma pessoa. 
$\mathrm{O}$ instrumento de pesquisa de mercado foi aplicado no mês de novembro de 2012, em população variada, ou seja, não se especificou um grupo particular para a coleta de dados. O objetivo foi obter uma amostra tão diversificada quanto possível, de forma a abranger diversas faixas etárias, graus de escolaridade, gênero e renda.

Foi elaborado um questionário contendo 31 perguntas fechadas, as quais os indivíduos deveriam responder escolhendo apenas uma alternativa; somente uma pergunta era aberta, na qual o respondente poderia dizer até quanto pagaria por um alimento com embalagem sustentável. Quatro perguntas tinham como objetivo a caracterização do respondente, e as restantes eram sobre conhecimento de meio ambiente, comportamento de compra e influência das práticas sustentáveis nas decisões de compra. A aplicação do instrumento foi feita via e-mail de forma aleatória, utilizando a ferramenta Google Docs. Após 30 dias do envio do instrumento, foi realizado o tratamento dos dados, avaliando a incidência de resposta e as médias para as 13 perguntas selecionadas.

As seis perguntas iniciais, após as de caracterização demográfica da amostra, tinham por objetivo delinear o comportamento de compra dos respondentes; a seguir os mesmos foram questionados quanto ao grau de conhecimento sobre sustentabilidade, produtos verdes, reciclagem e logística reversa. Essas perguntas permitiam que os respondentes avaliassem o próprio comportamento quanto às compras habituais e avaliassem se a sustentabilidade era considerada na hora de decidir por um produto. Por fim, o respondente deveria indicar sua probabilidade de compra de produtos sustentáveis ou não, ambos com custo médio de mercado ou $10 \%$ mais caro, além de responder até quanto aceitaria pagar por um alimento com embalagem sustentável.

\section{DESCRIÇÃO E ANÁLISE DOS RESULTADOS}

Oitenta e dois questionários foram considerados válidos e utilizados para a análise estatística da pesquisa. Participaram do estudo 31 homens e 51 mulheres. A distribuição etária entre os gêneros foi semelhante, sendo a faixa de 21 a 30 anos a de maior frequência (45\% para homens e 43\% para mulheres). Quanto à escolaridade, 
a maioria dos sujeitos possui graduação (39\% para homens e mulheres) ou pósgraduação (45\% para homens e 55\% para mulheres). Com relação à renda, a maioria afirmou ter renda superior a $\mathrm{R} \$ 5.000,00$ (61\% para homens e $49 \%$ para mulheres). De todos os respondentes, $81,7 \%$ são residentes em Porto Alegre (RS) ou região metropolitana e $18,3 \%$ do interior.

A Tabela 1 traz o número de repetição de resposta, a média e o desvio padrão para as perguntas relacionadas ao conhecimento sobre sustentabilidade e comportamento de compra, independente de gênero, idade ou escolaridade.

Tabela 1. Número de repetição de resposta, média e desvio padrão para conhecimento sobre sustentabilidade e comportamento de compra

\begin{tabular}{|c|c|c|c|c|c|c|c|}
\hline \multirow{2}{*}{ Conhecimento sobre sustentabilidade } & \multicolumn{5}{|c|}{ Incidência de resposta } & \multirow{2}{*}{ Média } & \multirow{2}{*}{$\begin{array}{l}\text { Desvio } \\
\text { padrão }\end{array}$} \\
\hline & 1 & 2 & 3 & 4 & 5 & & \\
\hline Me preocupo com o meio ambiente & 1 & 0 & 6 & 42 & 33 & 4,29 & 0,71 \\
\hline Possuo conhecimento sobre produtos verdes & 1 & 6 & 11 & 49 & 15 & 3,87 & 0,84 \\
\hline $\begin{array}{l}\text { Minha decisão de compra é voltada para } \\
\text { produtos com menor impacto ambiental }\end{array}$ & 5 & 15 & 20 & 37 & 5 & 3,27 & 1,03 \\
\hline $\begin{array}{l}\text { Minha decisão de compra é orientada pelo } \\
\text { preço }\end{array}$ & 13 & 37 & 24 & 8 & 0 & 2,33 & 0,86 \\
\hline $\begin{array}{l}\text { Minha decisão de compra é orientada } \\
\text { somente pela qualidade }\end{array}$ & 4 & 31 & 16 & 29 & 2 & 2,93 & 1,02 \\
\hline Raramente faço compras por impulso & 4 & 23 & 18 & 23 & 14 & 3,24 & 1,18 \\
\hline
\end{tabular}

1 - Discordo totalmente; 2 - Discordo; 3 - Nem discordo nem concordo; 4 - Concordo; 5 - Concordo totalmente.

Para as duas afirmações relacionadas ao conhecimento sobre sustentabilidade ("me preocupo com o meio ambiente" e "possuo conhecimento sobre produtos verdes"), a incidência maior de respostas se concentrou na opção que indica maior preocupação com o meio ambiente e conhecimento sobre produtos verdes. A maior incidência de resposta também se concentrou na opção "concordo" quando se perguntou se o entrevistado fazia a escolha de produtos que causassem menos impacto ambiental, demonstrando que a maioria dos respondentes, independente de gênero, idade e escolaridade, possui, ou alega possuir, conhecimento sobre sustentabilidade e tenta aplicá-lo no seu dia a dia. 
As afirmações "minha decisão de compra é orientada pelo preço" e "minha decisão de compra é orientada pela qualidade" visavam verificar qual era a percepção dos entrevistados quanto aos atributos mais importantes na hora de decidir pela compra de um produto. Apesar da maioria dos respondentes ter afirmado que suas decisões de compra não são orientadas pelo preço, 31 respondentes optaram por "discordo" e 29 por "concordo" quando questionados se suas compras eram influenciadas pelo atributo qualidade. As médias de 2,33 para a decisão de compra orientada pelo preço e 2,93 para decisão de compra orientada pela qualidade indicam que a maioria dos entrevistados respondeu entre "discordo" e "nem discordo nem concordo" nestas duas perguntas. Esse resultado sugere que tanto a qualidade do produto quanto o preço são fatores importantes na decisão e compra dos respondentes. Analisando as médias obtidas, também é possível inferir que a qualidade influencia mais na decisão do que o preço. A questão sobre compras por impulso obteve mesmo número de respostas tanto para "concordo" quanto para "não concordo"; ocorreram mais repetições para "concordo totalmente" do que para "discordo totalmente", indicando que a maioria dos respondentes acredita que não faça suas compras por impulso.

A seguir apresentaram-se quatro cenários para o respondente, através dos quais os sujeitos deveriam identificar o grau de sua intenção de compra. Avaliou-se primeiramente qual seria a intenção de compra de produtos com preço médio de mercado, sustentáveis ou não; a seguir, aumentou-se o preço dos dois produtos em relação ao preço médio de mercado, para verificar qual seria a influência do preço para produtos com embalagem sustentável ou não; as incidências, médias e desvio padrão estão na Tabela 2:

Tabela 2. Número de repetição de resposta, média e desvio padrão para os quatro cenários apresentados

(Continua)

\begin{tabular}{|c|c|c|c|c|c|c|c|}
\hline \multirow{2}{*}{ Grau de intenção de compra } & \multicolumn{5}{|c|}{ Incidência de resposta } & \multirow{2}{*}{ Média } & \multirow{2}{*}{$\begin{array}{l}\text { Desvio } \\
\text { padrão }\end{array}$} \\
\hline & 1 & 2 & 3 & 4 & 5 & & \\
\hline $\begin{array}{l}\text { Alimento com preço médio de mercado e } \\
\text { embalagem não sustentável }\end{array}$ & 12 & 27 & 34 & 9 & 0 & 2,49 & 0,88 \\
\hline $\begin{array}{l}\text { Alimento com preço médio de mercado e } \\
\text { embalagem sustentável }\end{array}$ & 1 & 0 & 8 & 54 & 19 & 4,10 & 0,66 \\
\hline
\end{tabular}


(Conclusão)

\begin{tabular}{|c|c|c|c|c|c|c|c|}
\hline \multirow{2}{*}{ Grau de intenção de compra } & \multicolumn{5}{|c|}{ Incidência de resposta } & \multirow{2}{*}{ Média } & \multirow{2}{*}{$\begin{array}{l}\text { Desvio } \\
\text { padrão }\end{array}$} \\
\hline & 1 & 2 & 3 & 4 & 5 & & \\
\hline $\begin{array}{l}\text { Alimento com preço } 10 \% \text { acima do preço de } \\
\text { mercado e embalagem não sustentável }\end{array}$ & 29 & 31 & 16 & 5 & 1 & 2,00 & 0,96 \\
\hline $\begin{array}{l}\text { Alimento com preço } 10 \% \text { acima do preço de } \\
\text { mercado e embalagem sustentável }\end{array}$ & 5 & 15 & 27 & 30 & 5 & 3,18 & 1,01 \\
\hline
\end{tabular}

1 - Muito baixa; 2 - Baixa; 3 - Nem baixa nem alta; 4 - Alta; 5 - Muito alta.

Dos 82 respondentes, 34 responderam que a probabilidade de compra de alimentos com preço médio de mercado e embalagem não sustentável não é nem baixa nem alta, com média 2,49 (entre "baixa" e "nem baixa nem alta"). Quando a embalagem é sustentável, 54 respondentes informaram que a probabilidade de compra era alta, resultado confirmado pela média de 4,10 (entre "alta" e "muito alta").

A rejeição da opção de alimento com preço $10 \%$ acima do preço de mercado e embalagem não sustentável também foi escolhida pela maioria dos entrevistados (média 2, com incidência de 31 respostas para a opção "baixa”). No caso da embalagem ser sustentável, a média obtida foi de 3,18 (entre "nem alta nem baixa" e "alta"), sendo que a incidência da resposta "alta" caiu em comparação com a opção de alimento com preço médio de mercado (30 para preço $10 \%$ acima do preço de mercado contra 54 para preço médio de mercado, ambas com embalagem sustentável).

Foi solicitado também que o respondente escolhesse entre dois produtos iguais, sendo um com embalagem não sustentável (A) e outro com embalagem sustentável (B). No cenário em que os dois produtos possuíam preços iguais, 80 dos 82 respondentes escolheram o produto B $(97,56 \%)$. Ao modificar o cenário, no entanto, em que B custava $10 \%$ mais do que A, a incidência de respostas mudou sensivelmente: de 80 foi para 49, representando 59,75\% do total de respondentes. Apesar desta diminuição da escolha pelo produto sustentável, mais da metade dos consumidores avaliados optaria pela embalagem sustentável, independente do preço ser mais alto ou não.

Existe um limite, no entanto, do quanto o consumidor aceitaria pagar a mais 
por um alimento com embalagem sustentável: 63 dos 82 entrevistados (76,83\%) responderam que não pagariam mais que $10 \%$ do preço de mercado por um alimento com embalagem sustentável, e a alternativa de pagar entre 11 e $20 \%$ acima do valor médio foi escolhida por apenas 14 respondentes, 17,07\%. Esse resultado coincide com o obtido na pergunta sobre compras orientadas somente pelo preço: a média foi de 2,33 , ou seja, entre "discordo" e "nem discordo nem concordo", com maior incidência na opção "discordo".

Estes resultados contribuem para responder o questionamento principal desta pesquisa, que é verificar se existe influência de práticas sustentáveis na decisão de compra do consumidor, neste caso, para alimentos com embalagens sustentáveis. Conclui-se que embalagens sustentáveis influenciam positivamente a intenção de compra, mas o acréscimo de preço que o consumidor está disposto a pagar por esse atributo é relativamente pequeno, oscilando em torno de 10\%. Quando os cenários foram analisados de forma estratificada, algumas diferenças foram observadas em relação às variáveis demográficas idade, escolaridade e renda, isto é, pessoas mais jovens, com maior grau de escolaridade e renda superior a $\mathrm{R} \$ 3.000,00$ apresentaram maior probabilidade para a compra de produtos alimentícios com embalagens sustentáveis. No entanto, essas diferenças caracterizam indicações preliminares, uma vez que os resultados referentes a cada categoria são baseados em amostras menores.

\section{CONSIDERAÇÕES FINAIS}

O investimento em embalagem sustentável para alimentos, seja essa embalagem biodegradável, reciclável ou que faz uso da logística reversa, é capaz de aumentar a intenção de compra dos consumidores em geral. Todavia, a intenção de compra diminuiu em todos os grupos quando o alimento com embalagem sustentável apresentava custo acima do preço de mercado (10\%), revelando que a tolerância a acréscimos no preço é pequena.

Uma vez que a cultura em que o indivíduo está inserido influencia no seu comportamento de compra, deve-se levar em consideração que o estudo foi realizado 
com amostragem aleatória de pessoas e pode não traduzir o comportamento real de brasileiros, em função das diferenças culturais existentes no país. Como 81,7\% dos respondentes são residentes em Porto Alegre ou região metropolitana, pode-se inferir que o comportamento registrado nesta pesquisa traduz o comportamento dos consumidores da capital gaúcha e regiões próximas.

Futuros estudos poderiam ser mais específicos quanto à amostra, selecionando pontos de venda e identificando o perfil dos consumidores que costumam frequentá-los. Os respondentes poderiam ser classificados por outros requisitos, além dos abordados neste estudo, como, por exemplo, estado civil, número de filhos, tipo de residência, estimativa dos custos semanais/mensais com alimentação - desde a alimentação durante o dia quanto gastos em supermercado - se costuma comprar alimentos prontos (como congelados) e com que frequência. Desse modo, poder-se-ia traçar o perfil do consumidor mais suscetível a adquirir alimentos cujas embalagens seguem práticas sustentáveis e, a partir destas informações, criar campanhas em pontos de venda diretamente voltadas para esse grupo, além do desenvolvimento de novos produtos. Essa abordagem permitiria também averiguar o motivo de certos grupos não serem afetados por campanhas de defesa do meio ambiente e traçar estratégias para sensibilizar essa parte da população a adquirir práticas mais sustentáveis.

\section{REFERÊNCIAS}

AKENJI, L.; BENGTSSON, M. Sustainable consumption and production in the Asia-Pacific region: effective responses in a resource constrained world. Kanagawa: IGES, 2010. (IGES White Paper, III). Cap. 2, p. 23-46.

ARAÚJO, A. T. de. Oportunidades de melhorias no gerenciamento de resíduos sólidos em uma indústria de plásticos: um estudo de caso. 2014. 136 f. Dissertação (Mestrado) - Universidade Estadual Paulista Júlio de Mesquita Filho, Faculdade de Engenharia, 2014. Disponível em: < http:/hdl.handle.net/11449/115590>. Acesso em: 2015. 
ao consumo sustentável na intenção de compra de produtos ecologicamente embalados. 2004. 159 f. Dissertação (Mestrado em Administração) - Universidade Federal do Rio Grande do Sul, Porto Alegre, RS.

BRANDALISE, L. T.; BERTOLINI, G. R. F.; ROJO, C. A.; LEZANA, A. G. R.; POSSAMAI, O. A percepção e o comportamento ambiental dos universitários em relação ao grau de educação ambiental. Gestão e Produção, São Carlos, v. 16, n. 2, p. 273-285, abr./ jun. 2009.

CARR, L. G. Desenvolvimento de embalagem biodegradável tipo espuma a partir de fécula de mandioca. 2007. 107 f. Dissertação (Doutorado em Engenharia Química) - Universidade de São Paulo, São Paulo, SP.

CHAVES, G. L. D.; BATALHA, M. O. Os consumidores valorizam a coleta de embalagens recicláveis? Um estudo de caso da logística reversa em uma rede de hipermercados. Gestão e Produção, v. 13, n. 3, p. 423-434, set./dez. 2006.

CLEVELAND, M.; KALAMAS, M.; LAROCHE, M. Shades of green: linking environmental locus of control and pro-environmental behaviors. Journal of Consumer Behavior, v. 22, n. 3, p. 198-212, 2005.

COUTINHO, B. C. et al. A importância e as vantagens do polihidroxibutirato (plástico biodegradável). Holos, v. 3, p. 76-81, 2004.

BRITO, M. P.; DEKKER, R. Reverse logistics: a framework. Econometric Institute, Report EI 2002-38, Erasmus University Rotterdam, The Netherlands, 2002.

ELKINGTON, J. Cannibals with Forks: the triple bottom line of 21st century business, Oxford: Capstone, 1997. 402 p.

FORLIN, F. S.; FARIA, J. A. F. Reciclagem de embalagens plásticas. Polímeros: ciência e tecnologia, v. 12, n. 1, p. 1-10, 2002.

GONÇALVES-DIAS, S. L. F.; TEODÓSIO, A. S. S. Estrutura da cadeia reversa: "caminhos" e "descaminhos" da embalagem PET. Produção, v. 16, n. 3, p. 429-441, set./dez. 2006. 
HAIR, J. F. et al. Análise multivariada de dados. [s.l.]: Bookman, 2007.

HISATUGO, E.; MARÇALJUNIOR, O. Coleta seletiva e reciclagem como instrumentos para conservação ambiental: um estudo de caso em Uberlândia, MG. Sociedade $\mathbf{e}$ Natureza, Uberlândia, v. 19, n. 2, p. 205-216, dez. 2007.

INSTITUTO AKATU. Responsabilidade social empresarial: percepção do consumidor brasileiro, São Paulo: [S.N.], 2004.

KOLLER, M.; FLOH, A.; ZAUNER, A. Further insights into perceived value and consumer loyalty: a green perspective. Psychology \& Marketing, v. 28, n. 12, p. 1154-1176, 2011.

MacDONALD, E. F.; SHE, J. Seven cognitive concepts for successful eco-design. Journal of Cleaner Production, v. 92, p. 23-36, 2015.

MALHOTRA, N. K. Pesquisa de marketing: uma orientação aplicada. [s.l.]: Bookman, 2006.

MOTTA, S. L. S. Motivações para o lançamento de um produto ecologicamente correto: um estudo de caso. Revista de Gestão USP, São Paulo, v. 14, n. 1, p. 31-40, jan./mar. 2007.

PARK, J.; HA, S. Understanding pro-environmental behavior: a comparison of sustainable consumers and apathetic consumers. International Journal of Retail and Distribution Management, v. 40, n. 5, p. 388-403, 2012.

PICKETT-BAKER, J.; OZAKI, R. Pro-environmental products: marketing influence on consumer purchase decision. Journal of Consumer Behavior, v. 25, n. 5, p. 281$293,2008$.

SICHE, R.; AGOSTINHO, F.; ORTEGA, E.; ROMEIRO, A. Índices versus indicadores: precisões conceituais na discussão da sustentabilidade de países. Ambiente e Sociedade, Campinas, v. 10, n. 2, p. 137-148, jul./dez 2007. 
WALDMAN, M. De onde vem o lixo produzido no mundo. Jornal Estadão, 28 de setembro de 2011. Disponível em: < http://www.estadao.com.br/infograficos/deonde-vem-o-lixo-produzido-no-mundo,sustentabilidade,235040>. Acesso em: 06 out. 2015.

WATKINS, L.; AITKEN, R.; MATHER, D. Conscientious consumers: a relationship between moral foundations, political orientation and sustainable consumption. Journal of Cleaner Production. Available online 18 June 2015.

WORLD COMMISSION ON ENVIRONMENT AND DEVELOPMENT. WCED. Our Common Future. Oxford, U.K.: Oxford University Press, 1987, 383 p.

ZHAO, H.; GAO, Q.; WU, Y.; WANG, Y.; ZHU, X. What affects green consumer behavior in China? A case study from Qingdao. Journal of Cleaner Production, v. 63, p. 143$151,2014$.

Recebido em: 15 de agosto de 2015 Revisado em: 17 de setembro de 2015 Aceito em: 22 de dezembro de 2015 